\title{
Conformational conversion and prion disease: authors' reply
}

\section{Mick F. Tuite and Tricia R. Serio}

In their correspondence to our article (The prion hypothesis: from biological anomaly to basic regulatory mechanism. Nature Rev. Mol. Cell Biol. 11, 823-833 (2010)), Shen and Ji (Conformational conversion and prion disease. Nature Rev. Mol. Cell Biol. 23 Mar 2011 (doi:10.1038/nrm3007-c1)) disagree with the central opinion that, in light of the emerging information on the phenotypic impact of fungal prions, we can no longer consider mammalian prion proteins as just infectious agents that cause disease. The concern raised by Shen and Ji, we believe, is largely a semantic one, and we hope that this response helps clear up any ambiguity that some readers of our article may have had.

In our Review, we suggest that switching to the prion form can result in a change of function for the protein that affects the host cell in some way. In the case of several fungal prions, this conformational conversion partially inactivates the protein and, because those factors normally act as regulators of basic cellular processes, these effects lead to global changes in gene expression and thereby cellular phenotype. However, the conformational conversion of a prion protein may also create new phenotypes through alternative mechanisms. As we discuss in our Review, the conformational conversion of other prion proteins in fungi endows them with new activities, with the associated cellular phenotypes reflecting a gain of function for the protein rather than a change in a regulatory network.

We did not mean to imply that, just because mammalian prion proteins (PrPs) and fungal prions propagate using a similar mechanism, $\operatorname{PrP}^{\mathrm{Sc}}$ is not an infectious agent; at no point in our article do we suggest that $\mathrm{PrPsc}^{\mathrm{Sc}}$ is anything other than linked with disease. Instead, we suggest that the infectivity and toxicity of $\mathrm{PrP}^{\mathrm{Sc}}$ are new 'functions' acquired by $\operatorname{PrP}^{\mathrm{C}}$. Indeed, fungal prions may also act as mediators of cellular toxicity in certain contexts, such as the overexpression of the yeast prion protein rich in Asn and Gln 1 (Rnq1) or a polyQ-expanded fragment of huntingtin in cells carrying the $\left[\mathrm{PIN}^{+}\right]$ prion form of Rnq1. Thus, switching to the prion form may be considered to be a way to regulate the function of a prion protein, whatever it may be.

Mick Tuite is at the Kent Fungal Group, School of Biosciences, University of Kent, Canterbury, Kent CT2 7NJ, UK.

Tricia Serio is at Brown University, Department of Molecular Biology, Cell Biology, and Biochemistry, Providence, Rhode Island 02912, USA. e-mails: M.F.Tuite@kent.ac.uk; TriciaSerio@Brown.edu doi: 10.1038/nrm3007-c2

Competing interests statement

The authors declare no competing financial interests. 